\title{
LE CONTENTIEUX DE CANDIDATURE À L'ÉLECTION DE GOUVERNEUR DE PROVINCE EN RÉPUBLIQUE DÉMOCRATIQUE DU CONGO. LE DOUBLE FONDEMENT DE LA COMPÉTENCE D'APPEL DU CONSEIL D'ÉTAT
}

\author{
Par KAHUNGA MAPELA Jean Jacques*
}

\section{Résumé}

La polémique née lors du traitement juridictionnel du contentieux de candidature des gouverneurs de provinces a été suscitée par l'exercice par certains candidats de leur droit constitutionnel de recours. Une certaine opinion a considéré que l'exercice de ce droit n'était pas fondé et que le Conseil d'Etat s'était arrogé des compétences qu'il n'avait pas étant donné qu'aux termes de l'article 27, alinéa 4 de la loi électorale, il est établi que le dispositif de l'arrêt ou du jugement qui est notifié aux parties concernées ainsi qu'à la Commission électorale n'est susceptible d'aucun recours.

Cette réflexion a montré que la polémique n'avait pas lieu d'être. Car, au-delà du fondement constitutionnel et légal évoqué par le Conseil d'Etat pour se reconnaitre cette compétence d'appel en la matière, les droits humains et l'Etat de droit sous-tendent également sa compétence d'appel. Ce qui signifie qu'en vertu du principe de la conformité à la Constitution de tous les actes infra constitutionnels, la loi électorale n'est pas fondée à déroger au droit de recours ainsi constitutionnalisé. Il s'en suit que la disposition de la loi électorale qui y déroge nécessite une réécriture pour éviter la cacophonie à laquelle ce contentieux a donné lieu.

\begin{abstract}
The controversy that arose during the jurisdictional handling of disputes concerning the candidature of provincial governors was prompted by the exercise by certain candidates of their constitutional right of appeal. A certain opinion considered that the exercise of this right was unfounded and that the Council of State had arrogated to itself powers that it did not have, given that under the terms of Article 27, paragraph 4 of the Electoral Law, it is
\end{abstract}

* Licencié agrégé en philosophie des ex Facultés Catholique de Kinshasa, actuellement Université Catholique du Congo (UCC), l'auteur de la présente étude est aussi licencié en droit public de l'Université de Kinshasa (UNIKIN). Il est chercheur au Centre de Recherches et d'Etudes sur l'Etat de Droit en Afrique (CREEDA) dans le département de la démocratie et de la participation citoyenne. 
established that the operative part of the judgment or judgement which is notified to the parties concerned and to the Electoral Commission is not subject to appeal.

This reflection showed that there was no need for controversy. For, beyond the constitutional and legal basis evoked by the Council of State to recognize its appellate jurisdiction in this matter, human rights and the rule of law also underlie its appellate jurisdiction. This means that by virtue of the principle of conformity with the Constitution of all infra-constitutional acts, the electoral law is not entitled to derogate from the right of appeal thus constitutionalized. It follows that the provision of the Electoral Act that derogates from it requires rewriting to avoid the cacophony to which this dispute has given rise.

\section{Introduction}

La gestion du contentieux de candidature de l'élection de gouverneur de province a soulevé une polémique. Ce fut un imbroglio politico-judiciaire ${ }^{1}$, justifié par la confusion à laquelle ce contentieux a donné lieu.

En effet, à l'issue du traitement des dossiers des candidatures, la Commission électorale nationale indépendante avait, par sa décision n³2/CENI/BUR/19 du 15 février 2019, déclaré recevables les candidats qui devraient concourir à cette élection des gouverneurs et vice-gouverneurs. Ainsi, deux candidatures avaient été retenues pour la province de Sankuru. Il s'était agi des messieurs Lambert Mende de la Convention des Congolais Unis (CCU), parti membre du Font Commun pour le Congo (FCC) et de Stéphane Mukumadi, candidat indépendant. Sur les cinq candidats de la province du sud Ubangi, celui de monsieur Tailla Nage Joachim n'avait pas été retenu. Sa candidature avait été jugée irrecevable.

Cette décision de validation des candidats de la Commission électorale se conformait aux prescrits de la loi électorale qui stipule que la Commission électorale nationale indépendante arrête et publie provisoirement les listes des candidats à la date fixée par elle. Et dans un délai de quatre jours suivant la publication de ces listes provisoires, les personnes concernées notamment le candidat dont l'éligibilité est contestée, le parti politique ou le regroupement politique ayant présenté un candidat ou une liste dans la circonscription et tout candidat se présentant individuellement sont habilitées à la mettre en cause ${ }^{2}$. Cette contestation a effectivement eu lieu à l'occasion des élections de gouverneur de province.

1 Stanis Bujakera Tshiamala, « RDC : Lambert Mende dans le Sankuru, une candidature unique qui tourne à l'imbroglio politico-judiciaire ", https://www.jeuneafrique.com/763518/politique/rdc-lamb ert-mende-dans-le-sankuru-une-candidature-unique-qui-tourne-a-limbroglio-politico-judicaire/, consulté le 01/10/2019.

2 Article 25 de la Loi n06/006 du 09 mars 2006 portant organisation des élections présidentielle, législatives, provinciales, urbaines, municipales et locales telle que modifiée par la Loi n ${ }^{\circ} 11 / 003 \mathrm{du}$ 25 juin 2011, la Loi n $15 / 001$ du 12 février 2015 et la Loi n ${ }^{\circ} 17 / 013$ du 24 décembre 2017 (texte coordonnés), JORDC, $59^{\mathrm{e}}$ année, Numéro spécial 7, juin 2018. 
Les décisions rendues par les juridictions concernées, à savoir la Cour administrative d'appel ${ }^{3}$, ont également été contestées, alors qu'elles ne devraient pas l'être, selon le législateur électoral qui a consacré l'insusceptibilité de recours ${ }^{4}$. Ce non recours consacré ne porte-t-il pas atteinte au droit de recours constitutionnellement garanti ? La décision du juge d'appel est-elle fondée sur la nécessité pour tout juge de garantir les libertés individuelles et les droits fondamentaux des citoyen, tel que celui d'être éligible?

Pour tenter de répondre à ces quelques préoccupations et, dans la perspective de la thématique de ce onzième séminaire relative à l'administration de la justice, cette étude qui analyse la façon dont le contentieux de candidature a été géré entend également répondre à la préoccupation de déterminer le juge compétent en la matière. Ainsi, tout en rappelant les faits de la cause ainsi que la polémique qui s'en est suivie (A), il importe d'apprécier l'argument du Conseil d'Etat fondant sa compétence d'appel. Au-delà du fondement constitutionnel et légal de cette compétence d'appel, celle-ci est également sous-tendue par le respect des droits de l'homme et de l'Etat de droit (B).

\section{A. Faits de la cause}

Pour ce qui est des faits de la cause, il convient de mettre en exergue deux faits majeurs, la contestation de la décision de l'instance électorale (I) et celle de l'œuvre du premier juge (II). Tout en indiquant la validité de ces contestations, il va falloir, en passant, effleurer la polémique qui a été alimentée du fait des décisions rendues par le Conseil d'Etat.

\section{Contestation de la décision de la Commission électorale}

Le contentieux de candidature est l'un des contentieux ${ }^{5}$ qui peut advenir lors du processus électoral. En tant que branche qui traite des litiges relatifs au processus électoral et vise à vérifier la régularité externe et interne d'un tel processus, ce contentieux peut se situer en amont de l'élection. Il est ainsi qualifié de contentieux pré-électoral. Ce qui signifie que la contestation qui en résulte peut surgir à l'occasion de divers actes, comme celui du dépôt des candidatures ${ }^{6}$.

Ce contentieux survient à la suite d'une décision de l'autorité compétente qui invalide ou valide les candidatures. Dans le cas de la RDC, il surgit à la suite de la décision de l'ins-

3 Article 27, al. 1 de la Loi n ${ }^{\circ} 06 / 006$ du 09 mars 2006 portant organisation des élections présidentielle, législatives, provinciales, urbaines, municipales et locales, note 2.

4 Article 27, al. 4 de la Loi n ${ }^{\circ} 06 / 006$ du 09 mars 2006 portant organisation des élections présidentielle, législatives, provinciales, urbaines, municipales et locales, note 2.

5 Michel Ilume Moke, Droit constitutionnel et Institutions politiques de la République Démocratique du Congo, Kisangani, 2018.

6 Francis Delpérée, «Le contentieux électoral », Annuaire international de justice constitutionnelle, 12-1996, 1997. L'école, la religion et la constitution- Constitution et élections, p.397. 
tance électorale qui arrête et publie les listes provisoires des candidats ${ }^{7}$. Comme on peut le constater, cette décision devient le fait générateur de ce contentieux qui nécessite pour son règlement l'implication de certaines autorités. Francis Delpérée parle des autorités politiques et administratives ${ }^{8}$. Dans le cas congolais, c'est l'autorité judiciaire qui est sollicitée.

L'autorité judiciaire est sollicitée pour contester la décision de la CENI. Dans le cas d'espèce, il s'est agi de la décision n³2/CENI/BUR/19 du 15 février 2019, déclarant recevables les listes des candidats indépendants, des partis et regroupements politiques à l'élection des gouverneurs et vice-gouverneurs. La décision ainsi contestée ouvrait la voie au recours des personnes concernées, étant donné que leur droit à l'éligibilité était mis en mal sinon en péril.

Au regard de la gravité de cette décision d'anéantir ce droit constitutionnel, il est nécessaire qu'elle soit la plus juste et la plus objective possible. C'est la raison pour laquelle il est exigé que les causes d'inéligibilité soient portées par la loi. Il s'agit d'éviter qu'une pareille décision soit teintée de relent de subjectivité. C'est à juste titre qu'en plus du traitement en vue de la sélection des candidatures opérée par l'administration électorale, la décision qu'elle prend est censurée par le juge. Ce qui permet d'assurer un double degré de traitement pour retenir ou rejeter une candidature à l'élection. Ce doublement traitement garantit, tant soit peu, la neutralité et l'objectivité.

C'est dans cette perspective qu'il faut inscrire la contestation de la décision de la commission électorale évoquée. Aux termes de la loi électorale, c'est dans un délai de quatre jours suivant la publication des listes provisoires que le candidat dont l'éligibilité est contestée, le parti politique ou le regroupement politique qui a présenté un candidat ou une liste dans la circonscription et tout candidat se présentant individuellement ${ }^{9}$ peuvent contester cette décision. Les décisions rendues par le juge compétent ont, à leur tour, été contestées sous forme d'appel devant le Conseil d'Etat.

\section{Contestation de l'œuvre du premier juge devant le Conseil d'Etat}

Les contestations enregistrées à l'occasion de cette élection trouvent dans la disposition électorale susvisée leur fondement et leur validité. Si la contestation portée par le candidat Tailla Nage Joachim entendait faire valoir son droit d'être éligible à cette fonction politique, droit que la décision de la CENI anéantissait, il n'en était pas le cas pour celle portée par le candidat Lambert Mende validé pour participer à cette élection. Ce dernier contestait, en revanche, cette décision pour avoir validé la candidature de son concurrent qu'il accusait

7 Article 25, al.1 de la de la Loi nº6/006 du 09 mars 2006 portant organisation des élections présidentielle, législatives, provinciales, urbaines, municipales et locales, note 2.

8 Francis Delpérée, «Le contentieux électoral », Annuaire international de justice constitutionnelle, note 6.

9 Article 25 de la Loi nº6/006 du 09 mars 2006 portant organisation des élections présidentielle, législatives, provinciales, urbaines, municipales et locales, note 2. 
détenir la double nationalité, en l'occurrence celle française ${ }^{10}$. Et, comme on le sait, nul n'est éligible s'il ne remplit la condition d'être notamment de la nationalité congolaise ${ }^{11}$, qui est une et exclusive en ce qu'elle ne peut être détenue concurremment avec une autre ${ }^{12}$.

Cette contestation étant fonction de type d'élections, eu égard à la répartition de compétence en la matière, celle de candidature à l'élection de gouverneur est dévolue au juge administratif, en l'occurrence la Cour administrative d'appe ${ }^{13}$. C'est cette juridiction, instituée par le constituant de $2006^{14}$ et organisée par le législateur organique, qui en a reçu la compétence ${ }^{15}$, notamment contentieuse ${ }^{16}$.

Relevons tout de même que le législateur organique ne parle que du contentieux des élections, là où le législateur électoral spécifie les types de contentieux, en contentieux des candidatures ${ }^{17}$, de campagne électorale ${ }^{18}$ et des résultats provisoires ${ }^{19}$. Et, lorsque le législateur électoral évoque, comme le législateur organique, le terme de contentieux des élections ${ }^{20}$, cette expression renvoie au contentieux des résultats. Doit-on comprendre le concept du contentieux des élections consacré par le législateur organique comme celui des résultats ou tel que spécifié par le législateur électoral, la préoccupation mérite qu'on s'y penche.

Cependant, au regard du régime qu'il lui est appliqué par ce législateur organique, il y a lieu de considérer que le concept de contentieux tel qu'il relève de ce législateur organique renvoie à tous les contentieux. Et, pour lui, quel que soit le type de contentieux, le double degré de juridiction est assuré. Cela étant, en matière contentieuse, comme c'est le cas pour

10 Stanis Bujakera Tshiamala, «RDC : Lambert Mende dans le Sankuru, une candidature unique qui tourne à l'imbroglio politico-judiciaire », note 1 .

11 Article 9 de la Loi n $06 / 006$ du 09 mars 2006 portant organisation des élections présidentielle, législatives, provinciales, urbaines, municipales et locales, note 2 .

12 Article 10, al.1 de la Constitution de la République Démocratique du Congo telle que modifiée par la Loi n ${ }^{\circ} 11 / 002$ du 20 janvier 2011 portant révision de certains articles de la République Démocratique du Congo du 18 février 2006 (textes coordonnées), JORDC, $59^{\mathrm{e}}$ année, Numéro spécial 7 , juin 2018.

13 Article 27, al.1 de la Loi nº6/006 du 09 mars 2006 portant organisation des élections présidentielle, législatives, provinciales, urbaines, municipales et locales, note 2.

14 Article 154 de la Constitution de la République Démocratique du Congo, note 12.

15 Article 155, al.4 de la Constitution de la République Démocratique du Congo, note 12.

16 Article 96 de la Loi Organique $\mathrm{n}^{\circ} 16 / 027$ du 15 octobre 2016 portant organisation, compétence et fonctionnement des juridictions de l'ordre administratif, JORDC, $57^{\mathrm{e}}$ année, numéro spécial, Kinshasa, 18 octobre 2016.

17 Article 27, al.5 de la Loi nº6/006 du 09 mars 2006 portant organisation des élections présidentielle, législatives, provinciales, urbaines, municipales et locales, note 2.

18 Article 35 de la Loi n06/006 du 09 mars 2006 portant organisation des élections présidentielle, législatives, provinciales, urbaines, municipales et locales, note 2.

19 Article 73 de la Loi nº6/006 du 09 mars 2006 portant organisation des élections présidentielle, législatives, provinciales, urbaines, municipales et locales, note 2 .

20 Article 74 de la Loi nº6/006 du 09 mars 2006 portant organisation des élections présidentielle, législatives, provinciales, urbaines, municipales et locales, note 2 . 
ce contentieux de candidature, le législateur organique garantit le recours à une instance supérieure à celle qui juge au premier degré. Ainsi, si la section du contentieux du Conseil d'Etat connait de l'appel des arrêts ainsi que des décisions rendues au premier ressort par des Cours administratives d'appel ${ }^{21}$, il en est autant pour les Cours administratives d'appel à propos des décisions rendues par les tribunaux administratifs ainsi que par des organes disciplinaires des provinces, des organismes publics ou des ordres professionnels provinciaux et locaux ${ }^{22}$. Et, en matière du contentieux des élections des gouverneurs et vice-gouverneurs de province, elle connait au premier degré de ce contentieux ${ }^{23}$. Ce qui sous-entend que la décision rendue est appelable. Elle l'est devant la section contentieuse du Conseil d'Etat.

Si le candidat Mukumadi a saisi cette haute juridiction administrative, c'était pour obtenir l'annulation de l'œuvre du premier juge de candidature qui avait invalidé sa candidature, alors qu'elle avait été retenue par l'instance électorale. La situation est tout autre pour le candidat Tailla Nage Joachim qui l'avait également saisi en appel, au regard de la décision du premier juge qui avait confirmé la décision d'invalidation de sa candidature prononcée par la commission électorale. Pour ce dernier candidat, il s'agirait d'une sorte de cassation vu que sa candidature avait été invalidée pour la première fois par la CENI avant que cette décision ne soit confirmée par la Cour d'appel de Gemena ${ }^{24}$.

Il s'en suit que le régime juridique consacré par ce législateur organique, concernant le contentieux de candidature, contredit celui institué par le législateur électoral, spécialisé dans la fixation des règles relatives à l'organisation des élections ${ }^{25}$ et, par conséquent, des contentieux qui adviennent. Pour le législateur spécialisé, la décision rendue par le tribunal compétent, à savoir la Cour administrative d'appel, n'est susceptible d'aucun recours. Il est établi, à cet effet, que « le dispositif de l'arrêt ou du jugement (...) n'est susceptible d'aucun recours $»^{26}$. Ce qui signifie que cette juridiction ne connaitrait pas au premier degré, mais plutôt, en premier et dernier ressort. Cela étant, en modifiant les listes de candidature,

21 Article 86 de la Loi organique ${ }^{\circ} 16 / 027$ du 15 octobre 2016 portant organisation, compétence et fonctionnement des juridictions de l'ordre administratif, note 16 .

22 Article 96 , al.1 de la Loi organique $n^{\circ} 16 / 027$ du 15 octobre 2016 portant organisation, compétence et fonctionnement des juridictions de l'ordre administratif, note 16 .

23 Article 96 , al.3 de la Loi organique $n^{\circ} 16 / 027$ du 15 octobre 2016 portant organisation, compétence et fonctionnement des juridictions de l'ordre administratif, note 16 .

24 «Le Conseil d'Etat annule l'élection du gouverneur du Sud-Ubangi »,https://www.radiookapi.net/ 2019/06/22/actualite/justice/le-conseil-detat-annule-lelection-du-gouverneur-du-sud-ubangi, consulté le 01/10/2019 ; " Sud-Ubangi : la Cour d'appel de Gemena rejette le recours du candidat Taila Nage », https://www.radiookapi.net/2019/04/22/actualite/justice/sud-ubangi-la-cour-dappel-d e-gemena-rejette-le-recours-du-candidat, consulté le 01/10/2019.

25 Article $1^{\text {er }}$ de la Loi nº6/006 du 09 mars 2006 portant organisation des élections présidentielle, législatives, provinciales, urbaines, municipales et locales, note 2.

26 Article 27, al.4 de la Loi nº6/006 du 09 mars 2006 portant organisation des élections présidentielle, législatives, provinciales, urbaines, municipales et locales, note 2. 
la Commission électorale arrêterait et publierait, sans délai, la liste définitive ${ }^{27}$ des candidats.

En agissant ainsi pour l'élection de gouverneur des provinces de sankuru et du sudubangi, la CENI entendait se conformer aux prescrits de la loi électorale. Mais, si cette élection a pu se tenir au sud-ubangi sans la participation de Tailla Nage Joachim, doublement invalidé, il n'en a pas été le cas pour le Sankuru où le Conseil d'Etat a tenu à l'application de sa décision réhabilitant la candidature de Mukamadi ${ }^{28}$, quoique controversée. En obtenant la suspension de l'élection du gouverneur de Sankuru à la date prévue ${ }^{29}$ et l'annulation $^{30}$ de celle du sud-Ubangi, le Conseil d'Etat semble avoir triomphé face à la polémique qu'avaient suscitée ses décisions de réhabilitation des candidats qui avaient fait appel des décisions d'invalidation de leurs candidatures par le premier juge.

Cette polémique était alimentée par la contradiction caractérisant le régime juridique applicable au contentieux de candidature. Face au Conseil d'Etat qui soutenait la légalité de sa compétence d'appel et l'application de toutes ces décisions, la CENI les qualifiait d'illégales $^{31}$. C'est pour cette raison qu'elle avait, malgré tout, programmé la tenue de cette élection du gouverneur de la province de Sankuru et, même, envisagé qu'elle se tienne sans tenir compte de l'atteinte du quorum ${ }^{32}$. Elle avait réussi à organiser celle de la province du Sud-Ubangi, sans la participation du candidat réhabilité par le Conseil d'Etat.

C'est dans cet ordre qu'il faut inscrire la division qui oppose le Conseil d'Etat au Conseil supérieur de la Magistrature qui s'écharpent sur la candidature de Joseph Mukumadi, invalidé par la Cour d'Appel de Lusambo pour défaut de citoyenneté congolaise, mais

27 Article 27, al.5 et 6 de la Loi n ${ }^{\circ} 06 / 006$ du 09 mars 2006 portant organisation des élections présidentielle, législatives, provinciales, urbaines, municipales et locales, note 2.

28 Innocent Olenga, «Au Conseil d'Etat : Mukumadi remis dans la course au Gouvernorat du Sankuru ! ", https://scooprdc.net/2019/03/27/au-conseil-detat-mukumadi-remis-dans-la-course-au-gouvernorat-du-sankuru/\#.XNwQA8gzbIU, consulté le 15/05/2019.

$29 J K M$, « RDC-Bataille Mende vs Mukumadi pour le gouvernorat du Sankuru : le conseil d'Etat ordonne la suspension de l'élection ", https://7sur7.cd/rdc-bataille-mende-vs-mukumadi-pour-legouvernorat-du-sankuru-le-conseil-detat-ordonne-la-suspension-de-lelection/, le 15/05/2019 ; Jules Mathieu Ekatou Limbele, Communiqué officiel du Conseil d'Etat ROR : 019, Kinshasa, 09 avril 2019.

30 « Le Conseil d'Etat annule l'élection du gouverneur du Sud-Ubangi », note 24 ; « Sud-Ubangi : " la décision du Conseil d'Etat est conforme et régulière ", affirme Pr Noel Botakile », https:// www.radiookapi.net/2019/06/23/actualite/politique/sud-ubangi-la-decision-du-conseil-detat-estconforme-et-regulier, consulté le 01/10/2019.

31 «Controverse autour de l'élection de gouverneur et vice-gouverneur du Sankuru, le président de la république calme le jeu ! », http://hautpanel.com/index.php/politique/item/2267-controverse-autour-de-l-election-de-gouverneur-et-vice-gouverneur-du-sankuru-le-president-de-la-republiquecalme-le-jeu, consulté le 01/10/2019.

32 « Controverse autour de l'élection de gouverneur et vice-gouverneur du Sankuru, le président de la république calme le jeu !», note 31 . 
validé par le Conseil d'Etat ${ }^{33}$. Si le Conseil supérieur de la Magistrature considère les arrêts du Conseil d'État comme "inexistants », et insusceptibles d'être exécutés, le Conseil d'Etat estime, de son coté, qu'ils restent valables. Et, pour lui, ils doivent être exécutés promptement au nom du président de la République, conformément aux dispositions pertinentes de la $l_{0 i}{ }^{34}$. Et, face à la menace d'ouverture d'action disciplinaire, proférée par le Bureau du Conseil supérieur de la Magistrature, contre les magistrats du conseil d'État ayant réhabilité Stéphane Mukumadi, le Conseil d'Etat a menacé de poursuites éventuelles toute personne ou institution qui se soustrairait à son obligation d'exécuter lesdits arrêts. Ces personnes et institutions seraient comptables de tout manquement préjudiciable en la matière ${ }^{35}$.

La base de cette polémique étant la contradiction relevée quant au régime juridique applicable au contentieux de candidature de l'élection de gouverneur de province, il va falloir analyser le fondement sur lequel le Conseil d'Etat base les arguments en faveur de sa compétence d'appel en la matière, avant d'envisager un autre soubassement.

\section{B. Fondement de l'argument du juge d'appel}

L'analyse de l'argument jurisprudentiel du juge d'appel pour déterminer sa compétence à connaitre, en dernier ressort, du contentieux de candidature pour l'élection du gouverneur de province permet d'en apprécier le fondement. Il s'agit d'élucider à la fois la légalité et la légitimité de cette compétence d'appel, au regard de la polémique entretenue autour de la gestion de ce contentieux.

Pour ce faire, il est impératif et indispensable d'exposer l'argument du juge concerné pour soutenir sa compétence d'appel. Il s'en suit, à cet effet, qu'en plus du fondement légal (I), cette compétence d'appel peut également être assise sur le double critère du respect des droits de l'homme et de la promotion de l'Etat de droit (II).

\section{Fondement légal : infériorité de la loi ordinaire face à la loi organique}

L'argument du Conseil d'Etat selon lequel la loi ordinaire ne peut primer sur la loi organique $^{36}$ a été développé pour soutenir sa compétence à connaitre au second degré du contentieux de candidature de l'élection de gouverneur de province. Il ne s'est pas agi d'un argument avancé pour réhabiliter les personnes qui l'avait ainsi saisie, mais pour justifier la

33 « Controverse autour de l'élection de gouverneur et vice-gouverneur du Sankuru, le président de la république calme le jeu ! », note 31 .

34 Bujakera Tshiamala, «RDC : Lambert Mende dans le Sankuru, une candidature unique qui tourne à l'imbroglio politico-judiciaire », note 1 .

35 Bujakera Tshiamala, «RDC : Lambert Mende dans le Sankuru, une candidature unique qui tourne à l'imbroglio politico-judiciaire », note 1 .

36 CE REA 002 du 27 mars 2019 Aff. Mukumadi Joseph Stéphane c/ Alliance, Guide électoral, Tome III, référencement jurisprudentiel 2006-2018, p. 296. 
contrariété de sa compétence d'appel face à l'insusceptibilité de tout recours consacrée par le législateur électoral.

Pour rappel, l'article 27 de la loi électorale identifie non seulement les juridictions compétentes pour connaitre du contentieux de candidature à différentes élections, il consacre également l'insusceptibilité de tout recours une fois que le juge ainsi identifié se serait prononcé sur ce contentieux.

Pour le Conseil d'Etat, qui entend fonder sa compétence d'appel en la matière, l'interdiction portée par cette disposition légale ne saurait lui être appliquée. En plus du fait qu'il tient cette compétence d'appel des articles de la Constitution et de la loi organique relative à son organisation et à son fonctionnement, la supériorité de cette dernière sur la loi ordinaire qui porte cette interdiction l'emporte. Ce qui implique sa détermination comme seule juridiction compétente chargée d'examiner, en appel, les arrêts rendus par les Cours d'Appel, juridictions de l'ordre judiciaire, statuant transitoirement sur les matières de la compétence des cours administratives d'appel ${ }^{37}$, celles-ci étant, affirme-t-il le juge compétent ${ }^{38} \mathrm{du}$ contentieux de candidature de l'élection de gouverneur de province.

Pour soutenir cette affirmation d'être cette juridiction compétente d'appel en la matière, le juge du Conseil d'Etat rappelle le fondement légal et constitutionnel de cette compétence. Il indique, à ce sujet que l'article 86 de sa loi organique le détermine. Car, cette disposition stipule que « la section contentieuse connait de l'appel des arrêts ainsi que des décisions rendus au premier ressort par des Cours administratives d'appel ». Cette disposition s'aligne sur l'article 155 de la Constitution qui dispose que sans préjudice des autres compétences que lui reconnait la Constitution ou la loi, « (...) Il connait en appel des recours contre les décisions des Cours administratives d'appel».

Ce doublement fondement constitutionnel et légal n'a pas suffi à ce juge pour asseoir et justifier cette compétence d'appel. Il l'a soutenu par cet autre argument de l'infériorité de la loi ordinaire sur celle organique. Cette infériorité ne pouvait lui interdire de connaitre du contentieux de candidature de l'élection de gouverneur de province au second degré. Pour en faire la démonstration, le juge d'appel rappelle que le fondement de la matière concernant le contentieux de candidature à l'élection du gouverneur de province n'est pas dans la disposition de l'article 27 de la loi électorale, mais dans l'article 165 de la même 1 loi $^{39}$. Cette dernière traite des réclamations et des contestations relatives à la validité d'une candidature à cette élection en y évoquant les délais de saisine et du prononcé qui sont respectivement de quarante huit heures et de sept jours.

Rien n'est rapporté, en revanche, sur cette interdiction de faire recours. Il en est autant de l'article 134 de la loi électorale auquel la disposition de l'article 165 renvoie pour ce qui est de la modalité de la publication de l'expédition de l'arrêt notifiée aux différents acteurs.

37 CE REA 002 du 27 mars 2019 Aff. Mukumadi Joseph Stéphane c/ Alliance, note 36.

38 Articles 27, al.1, 165 et 237bis, al.1 de la Loi n06/006 du 09 mars 2006 portant organisation des élections présidentielle, législatives, provinciales, urbaines, municipales et locales, note 2.

39 CE REA 002 du 27 mars 2019 Aff. Mukumadi Joseph Stéphane c/ Alliance, note 36. 
Cette autre disposition légale se contente à indiquer dans deuxième alinéa qu' " une décision dûment motivée est notifiée sans délai, à chaque candidat ou à son mandataire par la Commission électorale nationale indépendante ».

Par ailleurs, au constat qu'aucune interdiction de faire recours n'était indiquée par le siège de la matière, il soutient que chaque fois que le législateur a voulu interdire, il l'exprime par le renvoi y effectué. Dans le cas d'espèce, il n'en est rien. Ce qui implique que l'insusceptibilité de tout recours, tel qu'il en ressort de la disposition de l'article 27, al.4 de la loi électorale, ne saurait lui être appliquée, d'autant plus qu'elle ne vise pas spécialement les arrêts de la Cour administrative d'appel. Au contraire, pour lui, cette interdiction trouve sa place dans les contentieux électoraux portés directement devant la Cour constitutionnelle. C'est la raison pour laquelle la loi organique relative au fonctionnement de cette juridiction, texte supérieur à la loi électorale ordinaire, n'organise pas non plus les voies de recours. Se fondant ainsi sur cette infériorité de la loi électorale, qui est une loi ordinaire comparativement à la loi organique sur les juridictions de l'ordre administratif, cette interdiction d'insusceptibilité de tout recours ne saurait trouver application face à la consécration de l'appel de tous les arrêts et de toutes les décisions rendus au premier degré par les Cours administratives d'appel ${ }^{40}$.

Ainsi, lorsqu'une partie interjette devant lui appel de l'arrêt d'une Cour administrative d'appel statuant en matière de candidature, comme en matière de résultats, il est en même de s'en saisir. Il faut encore que l'appelant soit, au préalable, partie au procès au premier degré pour que son appel soit recevable. Le cas Joseph Stéphane Mukumadi en est illustratif autant que celui de Tailla Nage Joachim. L'argument du Conseil d'Etat qui vient d'être ainsi exposé pour justifier sa compétence d'appel pouvait également trouver son fondement en s'inscrivant sur le double critère des droits fondamentaux et sur la promotion de l'Etat de droit.

\section{Droits fondamentaux et promotion de l'Etat comme critères fondant le pouvoir d'appel du Conseil d'Etat}

La défense des droits de l'homme ainsi que la promotion de l'Etat de droit sont également le fondement justificatif de la compétence d'appel du Conseil d'Etat en matière de contentieux de candidature de l'élection de gouverneur de province.

En effet, le pouvoir judiciaire qui est indépendant des pouvoirs législatif et exécutif est dévolu aux cours et tribunaux dont le Conseil d'Etat ${ }^{41}$. Ce pouvoir judiciaire est établi comme garant des libertés individuelles et des droits fondamentaux ${ }^{42}$. Le Conseil d'Etat, à l'instar des autres juridictions de l'ordre administratif, ne peut s'y soustraire. Le recours

40 CE REA 002 du 27 mars 2019 Aff. Mukumadi Joseph Stéphane c/ Alliance, note 36.

41 Article 149, al.1 et 2 de la Constitution de la République Démocratique du Congo, note 12.

42 Article 150, al.1 de la Constitution de la République Démocratique du Congo, note 12. 
porté devant eux pour faire valoir la prétention à être éligible à la fonction de gouverneur de province doit être inscrit dans cette perspective.

Ainsi dit, si la compétence d'appel du Conseil d'Etat permet de garantir le droit à l'éligibilité, qui est un des aspects du droit de vote dont la nature politique est évidente, elle sert également la cause d'un autre droit constitutionnel garanti. Il s'agit du droit de former un recours, en l'occurrence, contre un jugement ${ }^{43}$. Si ce droit s'exerce dans les conditions fixées par la loi, il faut indiquer, à cet effet, que les tribunaux de grande instance, relevant de l'ordre judiciaire, ont compétence à connaitre de l'appel des jugements rendus par les tribunaux de paix ${ }^{44}$. Ils sont également susceptibles d'opposition ${ }^{45}$. Il en est de même pour les cours d'appel qui connaissent de l'appel des jugements rendus en premier ressort par les tribunaux de grande instance et les tribunaux de commerce ${ }^{46}$.

On ne peut rien en dire d'autre lorsque la Cour de cassation connait aussi de l'appel des arrêts rendus au premier degré par les Cours d'appel ${ }^{47}$ autant qu'elle est compétente pour connaitre des pourvois pour violation des traités internationaux dûment ratifiés, de la loi ou de la coutume contre des arrêts et jugements rendus en dernier ressort par les Cours et tribunaux civils et militaires ${ }^{48}$. La compétence de se pourvoir est même constitutionnelle. Ainsi, sans préjudice de ses autres compétences, la Cour de cassation connait des pourvois en cassation formés contre les arrêts et jugements rendus en dernier ressort par les Cours et tribunaux civils et militaires ${ }^{49}$.

L'exercice des voies de recours est également garanti par les juridictions de l'ordre administratif comme précédemment indiquer en parlant de la compétence en appel du Conseil d'Etat notamment. Car, pour les Cours administratives d'appel, cela ne fait aussi l'ombre d'aucun doute qu'elles connaissent, au second degré, pour leur section contentieuse, de l'appel des jugements et ordonnances rendus par les Tribunaux administratifs ainsi que des décisions prises par les organes disciplinaires des provinces, des organismes publics ou des ordres professionnels provinciaux et locaux ${ }^{50}$.

43 Article 21, al.2 de la Constitution de la République Démocratique du Congo, note 12.

44 Article 89, al.3 de la Loi n ${ }^{\circ}$ 3/011-B du 11 avril 2013 portant organisation, fonctionnement et compétence des juridictions de l'ordre judiciaire, JORDC, 59e année, Numéro spécial 7, juin 2018.

45 Article 88 de la Loi n $13 / 011$-B du 11 avril 2013 portant organisation, fonctionnement et compétence des juridictions de l'ordre administratif, note 44.

46 Article 91, al.1 de la Loi ${ }^{\circ} 13 / 011-\mathrm{B}$ du 11 avril 2013 portant organisation, fonctionnement et compétence des juridictions de l'ordre administratif, note 44 .

47 Article 94 de la Loi n $13 / 011-\mathrm{B}$ du 11 avril 2013 portant organisation, fonctionnement et compétence des juridictions de l'ordre administratif, note 44.

48 Article 95 de la Loi n 13/011-B du 11 avril 2013 portant organisation, fonctionnement et compétence des juridictions de l'ordre administratif, note 44 .

49 Article 153, al.2 de la Constitution de la République Démocratique du Congo, note 12.

50 Article 96, al.1 de la Loi n $16 / 027$ du 15 octobre 2016 portant organisation, compétence et fonctionnement des juridictions de l'ordre administratif, note 16. 
Il s'en suit donc que l'exercice du droit des recours tel qu'organisé par les différentes lois susmentionnées s'inscrit dans la perspective de la Constitution qui le garantit à tout celui qui estime que le jugement rendu l'aura été en sa défaveur. Ainsi, interdire l'exercice d'un tel droit, comme il en ressort de la disposition de l'article 27, al.4 de la loi électorale, c'est s'inscrire en porte à faux avec la Constitution et la promotion de l'Etat. Car, en établissant le contrôle de constitutionnalité, il s'agit de faire obligation aux actes infra-constitutionnels, tels que les lois, de se conformer à la Constitution. Il en est ainsi d'autant plus que tout acte qui serait déclaré non conforme à elle est nul de plein droit ${ }^{51}$.

Ainsi, si le Conseil d'Etat fondait également sa compétence d'appel sur la défense de ce droit, il ferait aussi œuvre utile comme s'il l'avait inscrit sur la perspective de l'Etat de droit. Car, comme on le sait, l'Etat de droit traduit l'aspiration de l'avènement d'une société gouvernée selon les règles de droit établies. Il s'agit d'une société respectueuse des droits de l'homme et des libertés des citoyens. Il s'est imposé comme le modèle idéal d'Etat qui fait « régner le droit » comme fondement et limitation du pouvoir ${ }^{52}$. Donnant un contenu minimum de l'Etat de droit, Ambroise Kamukuny Mukinay ${ }^{53}$ épingle notamment la hiérarchisation des actes du pouvoir. Ce qui permet de contrôler chaque acte en le rapportant aux actes qui lui sont supérieurs et le fait de s'adresser à un juge indépendant pour faire valoir ses prétentions, même contre l'Etat lui-même. Ce qui inclut l'exercice des voies des recours.

\section{Conclusion}

En annulant l'œuvre du premier juge pour mal jugé54, le Conseil d'Etat a affirmé sa compétence d'appel en ce qui concerne le contentieux de candidature de l'élection de gouverneur de province. Et, cela contre la compréhension selon laquelle l'arrêt du juge compétent en la matière, à savoir la Cour administrative d'appel, jugeait au premier et dernier ressort.

Cette étude l'a démontrée en fondant cette compétence du Conseil d'Etat sur les dispositions à la fois constitutionnelle et légale. En vertu de toutes ces dispositions, l'interdiction $\mathrm{du}$ non recours ne pourrait lier cette plus haute juridiction administrative en matière du contentieux de candidature de l'élection de gouverneur. Encore que cette interdiction est portée par la loi électorale, qui est une loi ordinaire et, de ce fait, inférieure à celle organique qui régit cette instance qui ne prévoit pas ce non recours, au contraire.

51 Article 168, al.2 de la Constitution de la République Démocratique du Congo, note 12.

52 Jean Chevallier, L'Etat de droit, $3^{\mathrm{e}}$ édition, Paris, Montchrestien, 1999, pp.127-128 cité par Cihunda Hengelela J., «Préface », p. ix, Hartmut Hamann, La conception allemande de l'Etat de droit et ses manifestations concrètes, op.cit, série de Conférences académiques du CREEDA, Publication n ${ }^{\circ} 001 / 2018$, Kinshasa, Octobre 2018.

53 Ambroise Kamukuny Mukinay, Contribution à l'étude de la fraude en droit constitutionnel congolais, Academia-L'Harmattan, Louvain-La-Neuve, 2011, p. 82 ; Ambroise Kamukuny Mukinay, Droit constitutionnel congolais, Editions Universitaires Africaines, Kinshasa, 2011, p. 41.

54 CE REA 002 du 27 mars 2019 Aff. Mukumadi Joseph Stéphane c/ Alliance, note 36. 
Ce fondement légal et constitutionnel illustre la meilleure administration de la justice en ce qui concerne ce contentieux, au-delà de la polémique suscitée par la saisine et les arrêts rendus par le Conseil d'Etat à cet effet. Au-delà de cet argument soutenu par ce juge pour décliner sa compétence d'appel en matière, il a été indiqué qu'en agissant ainsi, ce juge a servi la double cause des droits fondamentaux et de la promotion de l'Etat de droit. D'ailleurs, les droits fondamentaux dont le droit à l'éligibilité et celui de faire recours autant que la promotion de l'Etat de droit auraient également suffi pour fonder cette compétence.

\section{Bibliographie}

\section{Textes juridiques}

1. Constitution de la République Démocratique du Congo telle que modifiée par la Loi $n^{\circ}$ 11/002 du 20 janvier 2011 portant révision de certains articles de la République Démocratique du Congo du 18 février 2006 (textes coordonnées), JORDC, 59e année, Numéro spécial 7, juin 2018.

2. Loi nº6/006 du 09 mars 2006 portant organisation des élections présidentielle, législatives, provinciales, urbaines, municipales et locales telle que modifiée par la Loi $n$ ${ }^{\circ} 11 / 003$ du 25 juin 2011, la Loi n 15/001 du 12 février 2015 et la Loi n $17 / 013$ du 24 décembre 2017 (texte coordonnés), JORDC, 59e année, Numéro spécial 7, juin 2018.

3. Loi Organique $n^{\circ} 16 / 027$ du 15 octobre 2016 portant organisation, compétence et fonctionnement des juridictions de l'ordre administratif, JORDC, $57^{\mathrm{e}}$ année, numéro spécial, Kinshasa, 18 octobre 2016.

4. Loi n¹3/011-B du 11 avril 2013 portant organisation, fonctionnement et compétence des juridictions de l'ordre administratif, JORDC, $59^{\mathrm{e}}$ année, Numéro spécial 7 , juin 2018.

\section{Ouvrages}

1. Michel IlumeMoke, Droit constitutionnel et Institutions politiques de la République Démocratique du Congo, Presses Universitaires Patrice-Emery Lumumba de Kisangani, 2018.

2. Jean Chevallier, L'Etat de droit, $3^{\mathrm{e}}$ édition, Paris, Montchrestien, 1999, pp.127-128 cité par CihundaHengelela J., « Préface », p. ix, Hartmut Hamann, La conception allemande de l'Etat de droit et ses manifestations concrètes.

3. Ambroise KamukunyMukinay, Contribution à l'étude de la fraude en droit constitutionnel congolais, Academia-LHarmattan, Louvain-La-Neuve, 2011.

4. Ambroise KamukunyMukinay, Droit constitutionnel congolais, Editions Universitaires Africaines, Kinshasa, 2011. 
Sites internetconsultés

1. Stanis BujakeraTshiamala, «RDC : Lambert Mende dans le Sankuru, une candidature unique qui tourne à l'imbroglio politico-judiciaire », https://www.jeuneafrique.com/763 518/politique/rdc-lambert-mende-dans-le-sankuru-une-candidature-unique-qui-tourne-a -limbroglio-politico-judicaire/, consulté le 01/10/2019.

2. «Le Conseil d'Etat annule l'élection du gouverneur du Sud-Ubangi »,https://www.radi ookapi.net/2019/06/22/actualite/justice/le-conseil-detat-annule-lelection-du-gouverneur -du-sud-ubangi, consulté le 01/10/2019.

3. «Sud-Ubangi : la Cour d'appel de Gemena rejette le recours du candidat Taila Nage », https:/www.radiookapi.net/2019/04/22/actualite/justice/sud-ubangi-la-cour-dappel-degemena-rejette-le-recours-du-candidat, consulté le 01/10/2019.

4. InnocentOlenga, « Au Conseil d'Etat : Mukumadi remis dans la course au Gouvernorat du Sankuru ! », https://scooprdc.net/2019/03/27/au-conseil-detat-mukumadi-remis-dans -la-course-au-gouvernorat-du-sankuru/\#.XNwQA8gzbIU, consulté le 15/05/2019.

5. JKM, « RDC-Bataille Mende vs Mukumadi pour le gouvernorat du Sankuru : le conseil d'Etat ordonne la suspension de l'élection », https://7sur7.cd/rdc-bataille-mende-vs-mu kumadi-pour-le-gouvernorat-du-sankuru-le-conseil-detat-ordonne-la-suspension-de-lele ction/, consulté le 15/05/2019.

6. «Sud'Ubangi : " la décision du Conseil d'Etat est conforme et régulière », affirme Pr Noel Botakile »,https://www.radiookapi.net/2019/06/23/actualite/politique/sud-ubangi-1 a-decision-du-conseil-detat-est-conforme-et-regulier, consulté le 01/10/2019.

Autres

1. Jules Mathieu EkatouLimbele, Communiqué officiel du Conseil d'Etat ROR : 019, Kinshasa, 09 avril 2019.

2. CE REA 002 du 27 mars 2019 Aff. Mukumadi Joseph Stéphane c/ Alliance, Guide électoral, Tome III, référencement jurisprudentiel 2006-2018. 